\title{
Association of a polymorphism of BTN2A1 with dyslipidemia in East Asian populations
}

\author{
TETSUO FUJIMAKI ${ }^{1}$, KIMIHIKO KATO ${ }^{2}$, MITSUTOSHI OGURI $^{4}$, TETSURO YOHIDA ${ }^{1}$, HIDEKI HORIBE ${ }^{5}$, \\ KIYOSHI YOKOI ${ }^{5}$, SACHIRO WATANABE $^{6}$, KEI SATOH $^{7}$, YUKITOSHI AOYAGI $^{8}$, \\ MASASHI TANAKA ${ }^{8}$, HIROTO YOSHIDA ${ }^{9}$, SHOJI SHINKAI $^{9}$, YOSHINORI NOZAWA $^{10}$, \\ DONG-JIK SHIN ${ }^{11}$, JONG HO LEE ${ }^{11,12}$, YANGSOO JANG ${ }^{13}$ and YOSHIJI YAMADA ${ }^{3}$ \\ ${ }^{1}$ Department of Cardiovascular Medicine, Inabe General Hospital, Inabe; ${ }^{2}$ Meitoh Hospital, Nagoya and Life Science \\ Research Center, and ${ }^{3}$ Department of Human Functional Genomics, Life Science Research Center, Mie University, Tsu; \\ ${ }^{4}$ Department of Cardiology, Japanese Red Cross Nagoya First Hospital, Nagoya; ${ }^{5}$ Department of Cardiovascular Medicine, \\ Gifu Prefectural Tajimi Hospital, Tajimi; ${ }^{6}$ Department of Cardiology, Gifu Prefectural General Medical Center, Gifu; \\ ${ }^{7}$ Department of Vascular Biology, Institute of Brain Science, Hirosaki University Graduate School of Medicine, Hirosaki; \\ ${ }^{8}$ Department of Genomics for Longevity and Health, and ${ }^{9}$ Research Team for Social Participation and Health Promotion, \\ Tokyo Metropolitan Institute of Gerontology, Tokyo; ${ }^{10}$ Gifu International Institute of Biotechnology and \\ Tokai Gakuin University, Kakamigahara, Japan; ${ }^{11}$ Research Institute of Science for Aging; ${ }^{12}$ National \\ Research Laboratory of Clinical Nutrigenetics/Nutrigenomics, Department of Food and Nutrition, \\ College of Human Ecology, and ${ }^{13}$ Cardiology Division, Cardiovascular Center and Cardiovascular \\ Genome Center, College of Medicine, Yonsei University, Seoul, Republic of Korea
}

Received April 1, 2011; Accepted May 10, 2011

DOI: $10.3892 /$ etm.2011.266

\begin{abstract}
We previously identified rs6929846 of the butyrophilin, subfamily 2, member A1 gene (BTN2A1) as a susceptibility locus for myocardial infarction in Japanese individuals by a genome-wide association study. The aim of the present study was to examine the relation of the rs6929846 polymorphism of BTN2Al to dyslipidemia in Japanese and Korean populations, given that dyslipidemia is an important risk factor for myocardial infarction. A total of 10,953 individuals from three independent subject panels were examined. The relations of the rs6929846 polymorphism of BTN2Al to serum concentrations of triglycerides, high-density lipoprotein (HDL)-cholesterol and low-density lipoprotein (LDL)-cholesterol were examined in each subject panel. The $\mathrm{C} \rightarrow \mathrm{T}$ polymorphism (rs6929846) of BTN2Al was significantly associated with serum concentrations of triglycerides in Japanese subject panels A $(\mathrm{P}=0.0004)$ and $\mathrm{B}$ $(\mathrm{P}=0.0010)$, and in the Korean population $(\mathrm{P}=0.0095)$, with the minor $T$ allele being related to an increased serum concentration of triglycerides. The rs6929846 was associated
\end{abstract}

Correspondence to: Dr Yoshiji Yamada, Department of Human Functional Genomics, Life Science Research Center, Mie University, 1577 Kurima-machiya, Tsu, Mie 514-8507, Japan

E-mail: yamada@gene.mie-u.ac.jp

Key words: genetics, polymorphism, dyslipidemia, hyperlipidemia hypercholesterolemia with serum concentrations of HDL-cholesterol in Japanese subject panels A $(\mathrm{P}=0.0047)$ and $\mathrm{B}(\mathrm{P}=0.0015)$, with the $T$ allele being related to a decreased serum concentration of HDL-cholesterol, but not in the Korean population. This polymorphism was associated with the serum concentration of LDL-cholesterol only in Japanese subject panel B $(\mathrm{P}=0.0059)$, with the $T$ allele being related to an increased serum concentration of LDL-cholesterol. The results suggest that BTN2A1 may be a susceptibility gene for hypertriglyceridemia in East Asian populations and for low serum HDL-cholesterol in the Japanese population.

\section{Introduction}

Dyslipidemia is a multifactorial disorder caused by an interaction between genetic and environmental factors, the latter including a high-fat and high-calorie diet and physical inactivity $(1,2)$. Although recent genome-wide association studies (GWASs) have implicated several loci and candidate genes in predisposition to dyslipidemia in Caucasian populations (3-5), the genes that confer susceptibility to this condition in Asian populations remain to be identified definitively. We previously showed that the $\mathrm{C} \rightarrow \mathrm{T}$ polymorphism (rs6929846) of the butyrophilin, subfamily 2, member A1 gene (BTN2Al) is significantly associated with the prevalence of myocardial infarction in Japanese individuals by a GWAS (6). Given that dyslipidemia is an important risk factor for myocardial infarction, we hypothesized that the association of rs6929846 with myocardial infarction may be attributable, at least in part, to its effect on susceptibility to dyslipidemia. We thus examined 
the relation of rs6929846 of BTN2Al to dyslipidemia in Japanese and Korean populations.

\section{Patients and methods}

Study population. A total of 10,953 Japanese or Korean individuals from three independent subject panels was examined. The Japanese subject panel A comprised 3,319 individuals who either visited outpatient clinics of or were admitted to participating hospitals (Gifu Prefectural General Medical Center, Gifu; and Hirosaki University Hospital and Hirosaki Stroke Center, Hirosaki, Japan) between October 2002 and March 2009 because of various symptoms or for an annual health checkup. The Japanese subject panel B comprised 4,297 individuals who either visited outpatient clinics of or were admitted to participating hospitals (Gifu Prefectural Tajimi Hospital, Tajimi; Japanese Red Cross Nagoya First Hospital, Nagoya; and Inabe General Hospital, Inabe, Japan) between October 2002 and March 2009, or who were recruited to population-based cohort studies of aging and age-related diseases in Nakanojo, Kusatsu and Tokyo, Japan. The study protocol complied with the Declaration of Helsinki and was approved by the Committees on the Ethics of Human Research of Mie University Graduate School of Medicine, Hirosaki University Graduate School of Medicine, Gifu International Institute of Biotechnology, Tokyo Metropolitan Institute of Gerontology and participating hospitals. Written informed consent was obtained from each subject.

A total of 3,337 Korean subjects was drawn from the institutional patient databases of the Cardiovascular Genome Center and the Infarction Prognosis Study Registry, Severance Cardiovascular Hospital, Seoul. The study protocol complied with the Guidelines for Genome/Genetic Research issued by the Korean government and was approved by the Institutional Review Board of Yonsei University. Written informed consent was obtained from each participant.

Measurement of serum lipid profile. Venous blood was collected in the early morning after the subjects had fasted overnight and before they had begun lipid-lowering treatment as appropriate. Blood samples were centrifuged at $1,600 \mathrm{x} \mathrm{g}$ for $15 \mathrm{~min}$ at $4^{\circ} \mathrm{C}$, and serum was separated and stored at $-30^{\circ} \mathrm{C}$ until analysis. The serum concentrations of triglycerides, high-density lipoprotein (HDL)-cholesterol and low-density lipoprotein (LDL)-cholesterol were measured as previously described (7).

Genotyping of rs6929846 of BTN2A1. For Japanese individuals, venous blood $(7 \mathrm{ml})$ was collected into tubes containing ethylenediaminetetraacetic acid (disodium salt) at a final concentration of $50 \mathrm{mmol} / \mathrm{l}$, and genomic DNA was isolated with a kit (Genomix; Talent, Trieste, Italy). Genotype of rs6929846 of BTN2Al was determined at G\&G Science (Fukushima, Japan) by a method that combines the polymerase chain reaction (PCR) and sequence-specific oligonucleotide probes with suspension array technology (Luminex, Austin, TX, USA). Genotyping involved PCR amplification, hybridization, streptavidin-phycoerythrin reaction and measurement of fluorescence. Detailed genotyping methodology was described previously $(6,8)$.
For Korean subjects, genomic DNA was extracted from $5 \mathrm{ml}$ of whole blood with the use of a DNA isolation kit (WIZARD Genomic DNA purification kit; Promega, Madison, WI, USA). Genotype of rs6929846 was determined with the use of a TaqMan fluorogenic 5' nuclease assay (Applied Biosystems, Foster City, CA, USA). The PCR mixture (final volume, $5 \mu \mathrm{l}$ ) contained $2 \mathrm{ng}$ of genomic DNA, $2.5 \mu \mathrm{l}$ of TaqMan Universal PCR Master Mix and $0.125 \mu 1$ (or $0.25 \mu \mathrm{l}$ ) of 40X (or 20X) Assay Mix. The mixture was incubated first at $50^{\circ} \mathrm{C}$ for $2 \mathrm{~min}$ to activate uracil $\mathrm{N}$-glycosylase and to prevent carryover contamination, and then at $95^{\circ} \mathrm{C}$ for $10 \mathrm{~min}$ to activate DNA polymerase; it was subsequently subjected to 40 cycles of incubation at $92^{\circ} \mathrm{C}$ for $15 \mathrm{sec}$ and $60^{\circ} \mathrm{C}$ for $1 \mathrm{~min}$. All reactions were performed in 384-well plates with the use of a Dual 384-Well GeneAmp PCR System 9700 (Applied Biosystems), and endpoint fluorescence readings were performed with a PRISM 7900 HT Sequence Detection System (Applied Biosystems). Duplicate samples and negative controls were included to ensure accuracy of genotyping (6).

Statistical analysis. Quantitative data were compared between two groups by the unpaired Student's t-test. The Chi-square test was used to identify departures from Hardy-Weinberg equilibrium. A P-value of $<0.05$ was considered statistically significant. Statistical significance was examined by twosided test performed with JMP Genomics version 3.2 software (SAS Institute, Cary, NC, USA).

\section{Results}

The characteristics of the 10,953 subjects enrolled in the present study are shown in Table I. The prevalence of hypertriglyceridemia (a serum concentration of triglycerides of $\geq 1.65 \mathrm{mmol} / \mathrm{l}$ ), low-serum HDL-cholesterol (a serum concentration of HDL-cholesterol of $<1.04 \mathrm{mmol} / \mathrm{l}$ ) and high-serum LDL-cholesterol (a serum concentration of LDL-cholesterol of $\geq 3.64 \mathrm{mmol} / \mathrm{l}$ ) was $32.0,21.6$ and $25.7 \%$, respectively, in Japanese subject panel A; 37.0, 16.2 and $21.9 \%$, respectively, in Japanese subject panel B; and 28.0, 28.4 and 19.5\%, respectively, in the Korean population.

The relations of the BTN2A1 genotype to serum concentrations of triglycerides, HDL-cholestrol and LDL-cholesterol are shown in Table II. The $\mathrm{C} \rightarrow \mathrm{T}$ polymorphism (rs6929846) of BTN2Al was significantly $(\mathrm{P}<0.05)$ associated with the serum concentrations of triglycerides in Japanese subject panels A (dominant and recessive models) and B (dominant model), and in the Korean population (recessive model), with the minor $T$ allele being related to an increased serum concentration of triglycerides. The rs6929846 of BTN2Al was significantly associated with the serum concentrations of HDL-cholesterol in the Japanese subject panels A and B (dominant model), with the $T$ allele being related to a decreased serum HDL-cholesterol, but not in the Korean population. The rs6929846 of BTN2A1 was significantly associated with the serum concentrations of LDL-cholesterol in the Japanese subject panel B (recessive model), with the $T$ allele being related to an increased serum concentration of LDL-cholesterol, but not in the Japanese subject panel A or in the Korean population. In all Japanese and Korean individuals, rs6929846 of BTN2Al was significantly associated 
Table I. Characteristics of the 10,953 study subjects.

\begin{tabular}{lccc}
\hline \multirow{2}{*}{ Characteristics } & \multicolumn{2}{c}{ Japanese population } & Korean population \\
\cline { 2 - 3 } & Subject panel A & Subject panel B & \\
\hline No. of subjects & 3,319 & 4,297 & 3.337 \\
Age (years) & $65.1 \pm 11.40$ & $68.8 \pm 8.90$ & $59.6 \pm 10.30$ \\
Gender (male/female, \%) & $57.5 / 42.5$ & $58.3 / 41.7$ & $68.1 / 31.90$ \\
Body mass index $\left(\mathrm{kg} / \mathrm{m}^{2}\right)$ & $23.6 \pm 3.30$ & $23.5 \pm 3.40$ & $24.3 \pm 3.00$ \\
Current of former smoker $(\%)$ & 19.8 & 36.9 & 53.4 \\
Hypertension $(\%)$ & 61.5 & 67.3 & 65.0 \\
Diabetes mellitus $(\%)$ & 31.3 & 38.0 & 19.6 \\
Serum total cholesterol (mmol/l) & $5.15 \pm 1.00$ & $5.17 \pm 0.98$ & $4.77 \pm 1.02$ \\
Hypercholesterolemia $(\%)$ & 27.1 & 28.7 & 18.2 \\
Serum triglycerides (mmol/l) & $1.58 \pm 1.12$ & $1.68 \pm 1.12$ & $1.49 \pm 0.98$ \\
Hypertriglyceridemia $(\%)$ & 32.0 & 37.0 & 28.0 \\
Serum HDL-cholesterol (mmol/l) & $1.35 \pm 0.41$ & $1.40 \pm 0.40$ & $1.26 \pm 0.37$ \\
Low HDL-cholesterol (\%) & 21.6 & 16.2 & 28.4 \\
Serum LDL-cholesterol (mmol/l) & $3.11 \pm 0.88$ & $3.01 \pm 0.87$ & $2.85 \pm 0.91$ \\
High LDL-cholesterol (\%) & 25.7 & 21.9 & 19.5 \\
\hline
\end{tabular}

Data for age, body mass index and serum lipid concentrations are the means \pm SD. Hypertension: systolic blood pressure of $\geq 140 \mathrm{mmHg}$, diastolic blood pressure of $\geq 90 \mathrm{mmHg}$ or taking antihypertensive medication. Diabetes mellitus: fasting plasma glucose level of $\geq 6.93 \mathrm{mmol} / 1$, blood glycosylated hemoglobin content of $\geq 6.5 \%$ or taking antidiabetes medication. Hypercholesterolemia: a serum concentration of total cholesterol of $\geq 5.69 \mathrm{mmol} / \mathrm{l}$. Hypertriglyceridemia: a serum concentration of triglycerides of $\geq 1.65 \mathrm{mmol} / 1$. Low HDL-cholesterol: a serum concentration of HDL-cholesterol of $<1.04 \mathrm{mmol} / \mathrm{l}$. High LDL-cholesterol: a serum concentration of LDL-cholesterol of $\geq 3.64 \mathrm{mmol} / \mathrm{l}$.

with serum concentrations of triglycerides (dominant and recessive models), HDL-cholesterol (dominant model) and LDL-cholesterol (recessive model). Genotype distributions in the Japanese subject panels A and B and in the Korean population were all in Hardy-Weinberg equilibrium (Table II).

\section{Discussion}

Given that genetic factors and interactions between multiple genes and environmental factors are important in common forms of dyslipidemia (9), prediction of the risk for dyslipidemia on the basis of genetic variants would be beneficial for personalized prevention of this condition. We now showed that the $T$ allele of rs6929846 in BTN2Al was significantly associated with hypertriglyceridemia in Japanese and Korean populations, and with low-serum concentrations of HDL-cholesterol in Japanese individuals.

$B T N 2 A 1$ is a member of the cluster of butyrophilin genes (BTNs) (10). BTNs are type I membrane glycoproteins that were initially identified in bovine milk fat globules (11). $B T N s$ are located at the extended major histocompatibility complex region in chromosome 6 , constitute the immunoglobulin superfamily together with the $B 7$ gene family and butyrophilin-like gene (BTNL) family, and are widely expressed in a variety of human immune cells, suggesting a possible role in immune functions (12). Previous studies have shown significant relations of polymorphisms of BTNL2 with Kawasaki disease in Taiwanese children (13) and sarcoidosis in German (14) and American (15) populations, both of which are caused by inflammatory autoimmune responses.
We previously showed that the $T$ allele of rs6929846 was associated with an increased risk for myocardial infarction as well as with an increased transcription activity of BTN2Al (6). Overexpression of BTN2A1 decreased the expression of elastin mRNA and increased the mRNA expression of matrix metallopeptidase 3 and interleukin-5. In our preliminary experiment, the serum concentrations of high-sensitivity C-reactive protein were significantly greater in individuals in the combined group of $C T$ and $T T$ genotypes for rs6929846 of $B T N 2 A 1$ than in those with the $C C$ genotype in 755 healthy individuals without neoplastic, infectious or inflammatory disease (unpublished data). These observations suggest that the $T$ allele of rs6929846 of BTN2Al may accelerate inflammatory processes.

Inflammation is a key component of the regulation of lipid metabolism in rodents and primates (16). Inflammatory conditions under bacterial or viral infections were found to result in an increase in the serum concentration of triglycerides and a decrease in the serum HDL-cholesterol in humans $(17,18)$. Multiple cytokines are likely to affect the metabolism of cholesterol or triglycerides through several pathways, including the increased production and decreased clearance of very low-density lipoproteins, impaired reverse cholesterol transport and decreased excretion of bile acids (16,19-21). We now showed that rs6929846 of BTN2A1 was significantly associated with hypertriglyceridemia in Japanese and Korean populations and with reduced serum concentrations of HDL-cholesterol in Japanese individuals, with the minor $T$ allele representing a risk factor for these conditions. Acceleration of the inflammatory process by the $T$ allele of 


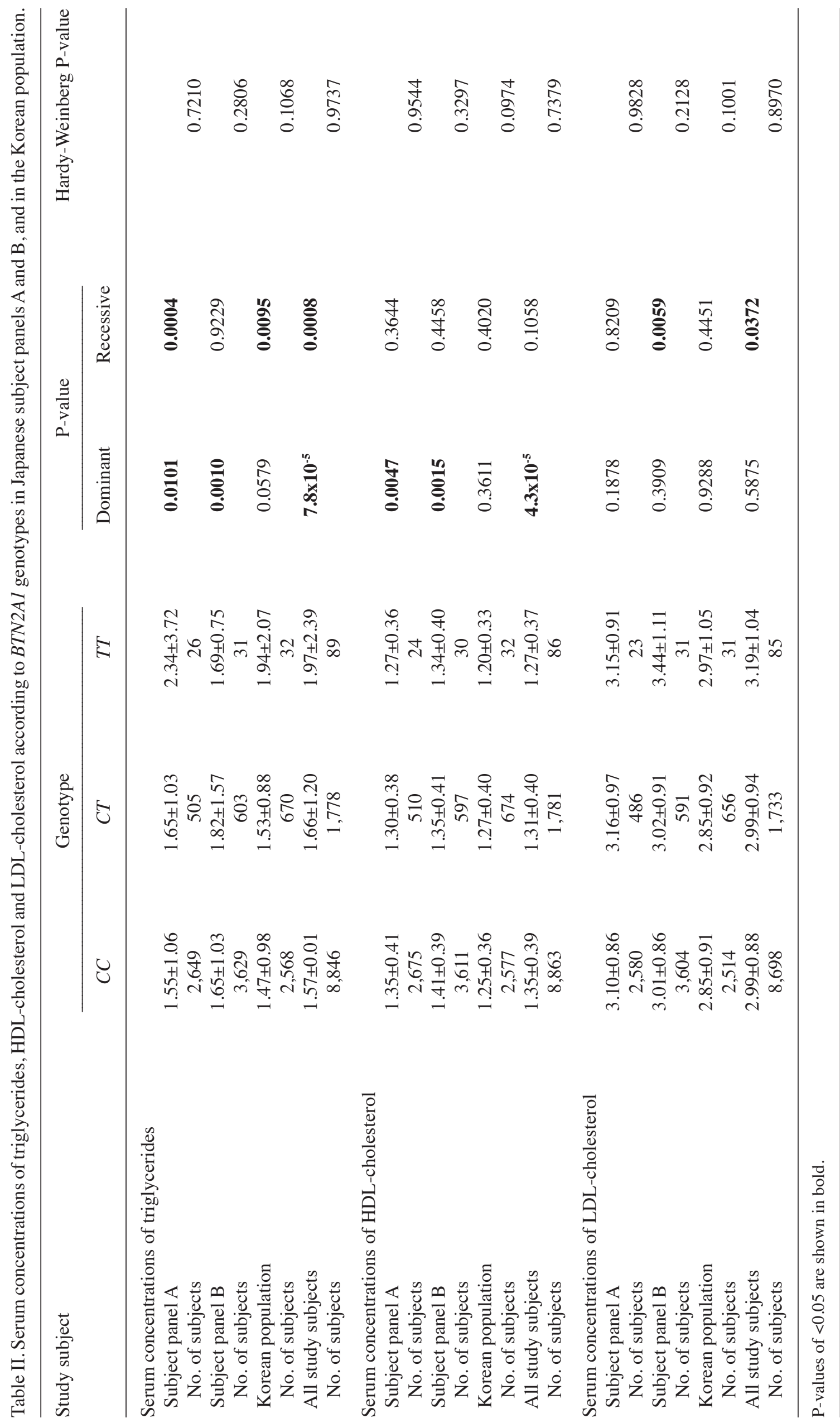


rs6929846 may result in alterations in lipid metabolism, although the underlying mechanism remains unknown.

There are limitations to the present study. i) Given that the study subjects were comprised only of Japanese and Korean individuals, validation of our findings is required in other ethnic groups. ii) It is possible that rs6929846 of BTN2A1 is in linkage disequilibrium with other polymorphisms in BTN2AI or in other nearby genes that are actually responsible for the development of dyslipidemia. iii) The functional relevance of rs6929846 of BTN2Al to the pathogenesis of dyslipidemia remains unknown. iv) Finally, environmental factors, such as dietary habits, that influence lipid metabolism were not evaluated in the present study.

In conclusion, BTN2A1 may be a susceptibility gene for hypertriglyceridemia in East Asian populations and for low-serum HDL-cholesterol in the Japanese population. Determination of genotypes for this polymorphism may prove informative for the assessment of the genetic risk for dyslipidemia in East Asian populations.

\section{Acknowledgements}

This study was supported by Grants-in-Aid for Scientific Research from the Ministry of Education, Culture, Sports, Science and Technology of Japan (no. 18209023, 18018021 and 19659149 to Y.Y.), and by a Research Grant from the Mie Medical Valley Project (to Y.Y.).

\section{References}

1. Khera AV and Rader DJ: Discovery and validation of new molecular targets in treating dyslipidemia: the role of human genetics. Trends Cardiovasc Med 19: 195-201, 2009.

2. Ordovas JM: Nutrigenetics, plasma lipids, and cardiovascular risk. J Am Diet Assoc 106: 1074-1081, 2006.

3. Aulchenko YS, Ripatti S, Lindqvist I, et al: Loci influencing lipid levels and coronary heart disease risk in 16 European population cohorts. Nat Genet 41: 47-55, 2009.

4. Kathiresan S, Melander O, Guiducci C, et al: Six new loci associated with blood low-density lipoprotein cholesterol, highdensity lipoprotein cholesterol or triglycerides in humans. Nat Genet 40: 189-197, 2008.
5. Kathiresan S, Willer CJ, Peloso GM, et al: Common variants at 30 loci contribute to polygenic dyslipidemia. Nat Genet 41: 56-65, 2009.

6. Yamada Y, Nishida T, Ichihara S, et al: Association of a polymorphism of BTN2A1 with myocardial infarction in East Asian populations. Atherosclerosis 215: 145-152, 2011.

7. Yamada Y, Matsuo H, Warita S, et al: Prediction of genetic risk for dyslipidemia. Genomics 90: 551-558, 2007.

8. Itoh Y, Mizuki N, Shimada T, et al: High-throughput DNA typing of HLA-A, -B, -C, and -DRB1 loci by a PCR-SSOP-Luminex method in the Japanese population. Immunogenetics 57: 717-729, 2005.

9. Garg A and Simha V: Update on dyslipidemia. J Clin Endocrinol Metab 92: 1581-1589, 2007

10. Rhodes DA, Stammers M, Malcherek G, Beck S and Trowsdale J: The cluster of BTN genes in the extended major histocompatibility complex. Genomics 71: 351-362, 2001.

11. Heid HW, Winter S, Bruder G, Keenan TW and Jarasch ED: Butyrophilin, an apical plasma membrane-associated glycoprotein characteristic of lactating mammary glands of diverse species. Biochim Biophys Acta 728: 228-238, 1983.

12. Arnett HA, Escobar SS and Viney JL: Regulation of costimulation in the era of butyrophilins. Cytokine 46: 370-375, 2009.

13. Hsueh KC, Lin YJ, Chang JS, Wan L and Tsai FJ: BTNL2 gene polymorphisms may be associated with susceptibility to Kawasaki disease and formation of coronary artery lesions in Taiwanese children. Eur J Pediatr 169: 713-719, 2010.

14. Valentonyte R, Hampe J, Huse K, et al: Sarcoidosis is associated with a truncating splice site mutation in BTNL2. Nat Genet 37: 357-364, 2005.

15. Rybicki BA, Walewski JL, Maliarik MJ, Kian $\mathrm{H}$ and Iannuzzi MC: The BTNL2 gene and sarcoidosis susceptibility in African Americans and Whites. Am J Hum Genet 77: 491-499, 2005.

16. Khovidhunkit W, Kim MS, Memon RA, et al: Effects of infection and inflammation on lipid and lipoprotein metabolism: mechanisms and consequences to the host. J Lipid Res 45: 1169-1196, 2004

17. Gallin JI, Kaye D and O'Leary WM: Serum lipids in infection. N Engl J Med 281: 1081-1086, 1969.

18. Sammalkorpi K, Valtonen V, Kerttula Y, Nikkila E and Taskinen MR: Changes in serum lipoprotein pattern induced by acute infections. Metabolism 37: 859-865, 1988.

19. Chen X, Xun K, Chen L and Wang Y: TNF-alpha, a potent lipid metabolism regulator. Cell Biochem Funct 27: 407-416, 2009

20. McGillicuddy FC, de la Llera Moya M, Hinkle CC, et al: Inflammation impairs reverse cholesterol transport in vivo. Circulation 119: 1135-1145, 2009.

21. Reiss AB: Effects of inflammation on cholesterol metabolism: impact on systemic lupus erythematosus. Curr Rheumatol Rep 11: 255-260, 2009. 